\title{
Interleukin-6-dependent survival of multiple myeloma cells involves the Stat3- mediated induction of microRNA-21 through a highly conserved enhancer
}

\author{
Dennis Löffler ${ }^{1,5^{*}}$, Katja Brocke-Heidrich ${ }^{1,5 *}$, Gabriele Pfeifer $^{1}$, Claudia Stocsits ${ }^{2}$, \\ Jörg Hackermüller ${ }^{3}$, Antje K. Kretzschmar ${ }^{3}$, Renate Burger ${ }^{4}$, Martin Gramatzki ${ }^{4}$, \\ Conny Blumert $^{1}$, Kay Bauer ${ }^{1,5}$, Helena Cvijic ${ }^{1}$, A. Kerstin Ullmann ${ }^{1}$, Peter F. Stadler ${ }^{2,3}$ \\ and Friedemann Horn ${ }^{1,3,58}$
}
${ }^{1}$ Institute of Clinical Immunology and Transfusion Medicine, Medical Faculty, University of Leipzig, 04103 Leipzig, Germany; ${ }^{2}$ Bioinformatics Group, Department of Computer Science, University of Leipzig, 04107 Leipzig, Germany; ${ }^{3}$ Fraunhofer Institute of Cell Therapy and Immunology, 04103 Leipzig, Germany; ${ }^{4}$ Division of Stem Cell Transplantation and Immunotherapy, 2nd Medical Department, University Hospital Schleswig-Holstein Campus Kiel, 24105 Kiel, Germany; ${ }^{5}$ Interdisciplinary Center for Clinical Studies, University of Leipzig, 04103 Leipzig, Germany

* These authors contributed equally to this work.

$\S$ Address all correspondence to: Prof. Dr. Friedemann Horn, Institute of Clinical Immunology and Transfusion Medicine, University of Leipzig, Johannisallee 30, 04103 Leipzig, Germany. Phone: +49 (341) 972-5491, Fax: +49 (341) 972-5509 email: friedemann.horn@medizin.unileipzig.de

Subject category: Neoplasia

Running Title: Regulation of microRNA-21 expression by Stat3

Total text word count: 1390

Abstract word count: 99 


\begin{abstract}
Signal transducer and activator of transcription 3 (Stat3) is implicated in the pathogenesis of many malignancies and essential for IL-6-dependent survival and growth of multiple myeloma cells. Here, we demonstrate that the gene encoding oncogenic microRNA-21 (miR-21) is controlled by an upstream enhancer containing two Stat3 binding sites strictly conserved since the first observed evolutionary appearance of miR-21 and Stat3. MiR-21 induction by IL-6 was strictly Stat3-dependent. Ectopically raising miR-21 expression in myeloma cells in the absence of IL-6 significantly reduced their apoptosis levels. These data provide strong evidence that miR-21 induction contributes to the oncogenic potential of Stat3.
\end{abstract}

Corresponding author's email address: friedemann.horn@medizin.uni-leipzig.de 


\section{Introduction}

Stat3, the major mediator of IL-6 signaling, ${ }^{1,2}$ participates in cellular transformation and oncogenesis. ${ }^{3,4}$ Its activation is essential for the transforming potential of many oncogenes. Previously, we could show that Stat3 activation is required for IL-6-dependent growth and survival of the human myeloma cell lines INA-6 and XG-1. ${ }^{5}$ Although microarray analyses revealed that in these cells the vast majority of IL-6 target genes is controlled through Stat3, differential expression of known apoptosis regulators, e.g. members of the Bcl-2 family, could not sufficiently account for the observed Stat3-mediated survival effect. ${ }^{5}$ We therefore searched for additional mechanisms through which Stat3 may suppress apoptosis in cancer cells.

MicroRNAs (miRNAs) are an abundant class of short non-protein-coding RNAs (ncRNAs) mediating posttranscriptional down-regulation of target genes. They have been shown to play a fundamental role in diverse physiological and pathological processes including proliferation, apoptosis, differentiation, and cell fate determination. ${ }^{6,7}$ Recent evidence indicates that miRNAs can function as tumor suppressors and oncogenes. ${ }^{8}$ Hence, we reasoned that miRNAs might be implicated in the Stat3-mediated survival of myeloma cells.

\section{Materials and Methods}

A detailed description of the bioinformatic procedures and the vectors and primer sequences used is given as supplemental material.

INA-6 and XG-1 myeloma and HepG2 hepatocellular carcinoma cells were cultivated and reporter gene assays performed as recently described. ${ }^{5,9}$

Chromatin immunoprecipitation was performed using a kit (EZ ChIP) from Upstate (Lake Placid, NY, USA) according to the manufacturer's instructions. Co-immunoprecipitated DNA was amplified using primers specific for the miR-21 upstream region and analysed on an agarose gel. Anti-Stat3 serum (PA-ST3) was from R\&D Systems (Wiesbaden, Germany).

RNA interference knock-down of Stat3 and overexpression of miR-21 in myeloma cells was performed by electroporation with pSUPER vectors as described. ${ }^{9}$ Shortly, $1 \mu \mathrm{g}$ of green fluorescence protein expression vector pEGFP-N1 (Clontech, Mountain View, CA, USA) and 19 $\mu \mathrm{g}$ of expression vectors encoding either a small hairpin RNA targeting Stat3 mRNA (pSUPERsiStat3) ${ }^{9}$ or precursor miR-21 (pSUPER-miR-21), or a pSUPER control vector were electroporated into $5 \times 10^{6}$ cells. For each experiment, $3 \times 10^{7}$ cells were combined and 
successfully transfected, green fluorescent cells sorted $48 \mathrm{~h}$ posttransfection using a FACSVantage Sorter (BD Biosciences, Heidelberg, Germany).

For real-time PCR of Stat3 mRNA and primary miR-21 transcripts (pri-miR-21), total RNA was prepared using the mirVana miRNA Isolation Kit (Ambion, Austin, TX, USA) and quantified by the RNA 6000 LabChip Kit (2100 Bioanalyzer, Agilent Technologies, Waldbronn, Germany). Reverse transcription was done with the SuperScript III First-Strand Synthesis System for RTPCR (Invitrogen, Karlsruhe, Germany). Real-time PCR was performed using a LightCycler (Roche, Mannheim, Germany). An optimal PCR reaction was established with the FastStart DNA Master SYBR Green I Kit (Roche, Mannheim, Germany), and it was taken care that only one PCR product was generated. Specificity of amplification was validated by confirming the identity of PCR products by sequencing. Mature miR-21 was detected by stem-loop reverse transcription followed by real-time PCR. ${ }^{10}$ Signals were normalized to values obtained for glyceraldehyde-3phosphate dehydrogenase.

Apoptosis was detected by flow-cytometry using annexin-V staining as previously described. ${ }^{5}$

\section{Results and Discussion}

To investigate whether Stat3 is involved in the regulation of miRNA genes, we analysed the genomic distribution of Stat3 binding sites and their correlation with known miRNAs by phylogenetic footprinting (for details, see supplemental material). Among the miRNA genes associated with evolutionarily conserved putative Stat 3 binding sites, $m i R-21$ has been described as an oncogenic miRNA exhibiting antiapoptotic activity in various carcinomas. ${ }^{11-13}$ The putative regulatory region of the $m i R-21$ gene is located within an intron of the overlapping transmembrane protein 49 (TMEM49) gene and contains two consensus Stat3 binding sites approximately $800 \mathrm{bp}$ upstream of the transcription start site. It is evident from Fig. 1A that the Stat 3 sites and the surrounding region, spanning $300 \mathrm{bp}$, are highly conserved in vertebrates at least since the common ancestor of tetrapods and actinopterygian fish, i.e. since the first evolutionary appearance of $m i R-21$ and Stat3 in species for which genome-wide data are available. 


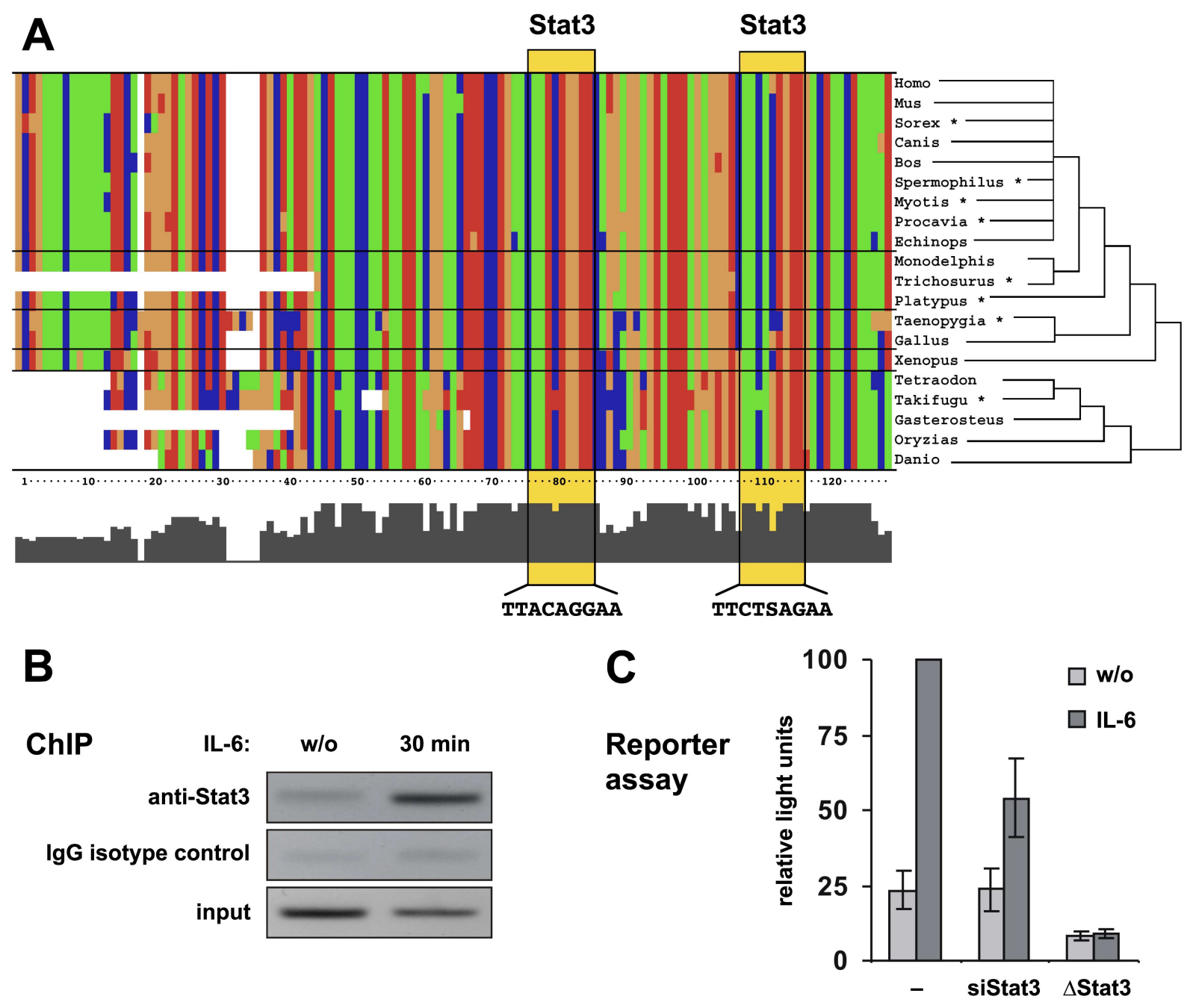

Fig. 1. A highly conserved enhancer is responsible for the Stat3-mediated responsiveness of the miR-21 promoter to IL-6. (A) 130-bp regions containing two predicted Stat3 binding sites upstream of the miR-21 genes of various vertebrate species are aligned. T, G, C, and A nucleotides are coloured green, orange, blue and red, respectively. Stat3 sites are highlighted by yellow boxes. Sequences from organisms where no genomic sequence was available were obtained from the Ensembl trace repository (as marked with a star, see supplemental Table S2). (B) XG-1 cells were deprived of IL-6 for 72 hours or restimulated for $30 \mathrm{~min}$ and subjected to a ChIP assay using anti-Stat3 or IgG isotype control. Co-immunoprecipitated DNA was amplified by PCR with primers specific for the miR-21 upstream enhancer. (C) Reporter gene assays were performed in HepG2 cells transfected either with a luciferase vector driven by the miR-21 promoter/enhancer alone (-) or in the presence of a vector encoding a small hairpin RNA silencing Stat3 expression (siStat3), or with a miR-21 promoter/enhancer construct containing point mutations in both Stat3 sites ( $\Delta$ Stat3). Values represent the mean luciferase activities \pm standard deviation of 3 independent experiments relative to samples from unmanipulated IL-6-treated cells.

Genomic data suggest that $m i R-21$ and its associated Stat3 binding sites are functionally independent of TMEM49 (supplemental Table S1). A stable distance between $m i R-21$ and the Stat3 sites throughout all vertebrates strongly suggests their functional correlation (supplemental Fig. S1). 
Chromatin immunoprecipitation revealed that Stat3 is indeed recruited to the miR-21 upstream region in XG-1 human myeloma cells in response to IL-6 (Fig. 1B). Furthermore, a genomic DNA fragment extending from -1120 to +25 bp relative to the miR-21 transcription start site ${ }^{14}$ and containing the putative Stat3 enhancer region proved to be IL-6-responsive in a reporter assay (Fig. 1C). IL-6-induced miR-21 promoter activity was abolished when the Stat3 motifs were eliminated by site-directed mutagenesis. Likewise, knocking down Stat3 by RNA interference inhibited miR-21 promoter activity. The effects of Stat3 knockdown could be rescued by expression of an siRNA-resistant variant of Stat3 (supplemental Fig. S2). These data demonstrate the existence of a functional Stat3-regulated enhancer upstream of the miR-21 gene, as predicted by the bioinformatic analysis.

To verify that IL-6 induces the transcription of the miR-21 gene, primary miR-21 transcripts (primiR-21) and mature miR-21 were quantified by real-time PCR in XG-1 and INA-6 myeloma cells. IL-6 increased pri-miR-21 expression substantially in either cell line (Fig. 2A). Upon Stat3 knock-down, comparable reductions of Stat3 mRNA and pri-miR-21 levels were observed (Fig. 2B) proving that miR-21 induction by IL-6 is mediated by Stat3. Induction of miR-21 expression by IL-6 is not restricted to multiple myeloma as it is observed in other tumor cell lines as well (supplemental Fig. S3). Taken together, our data demonstrate that miR-21 gene transcription is controlled by IL-6 and requires Stat3. In contrast to the rapid induction of pri-miR-21, mature miR-21 levels raised slowly in XG-1 cells, eventually reaching a 4-fold increase (Fig. 2C). The same was observed in prostate carcinoma cells (supplemental Fig. S3) indicating a rather slow processing rate of pri-miR-21.

Since miR-21 is involved in the control of tumor cell apoptosis ${ }^{11-13}$ we reasoned that it might contribute to the Stat3-dependent survival of myeloma cells. Due to relatively high basal miR-21 expression levels, a miR-21 knock-down might cause effects that go beyond Stat 3 regulation. Therefore, we chose to ectopically elevate miR-21 expression in the absence of IL-6 to levels comparable to those observed in response to Stat3 activation. Upon electroporation of INA-6 cells with a miR-21 expression vector and sorting the transfected cells, we determined a 2.4-fold increase in mature miR-21 expression over endogenous levels by real-time PCR analysis (mean of three independent experiments). As reported earlier, ${ }^{5}$ IL-6 withdrawal rapidly induced apoptosis in INA-6 cells (Fig. 2D). Raising miR-21 expression, however, yielded significantly higher levels of non-apoptotic cells (Fig. 2E). This effect was most dramatic in the absence of IL-6, reducing the cytokine-dependency of the INA-6 cells. 

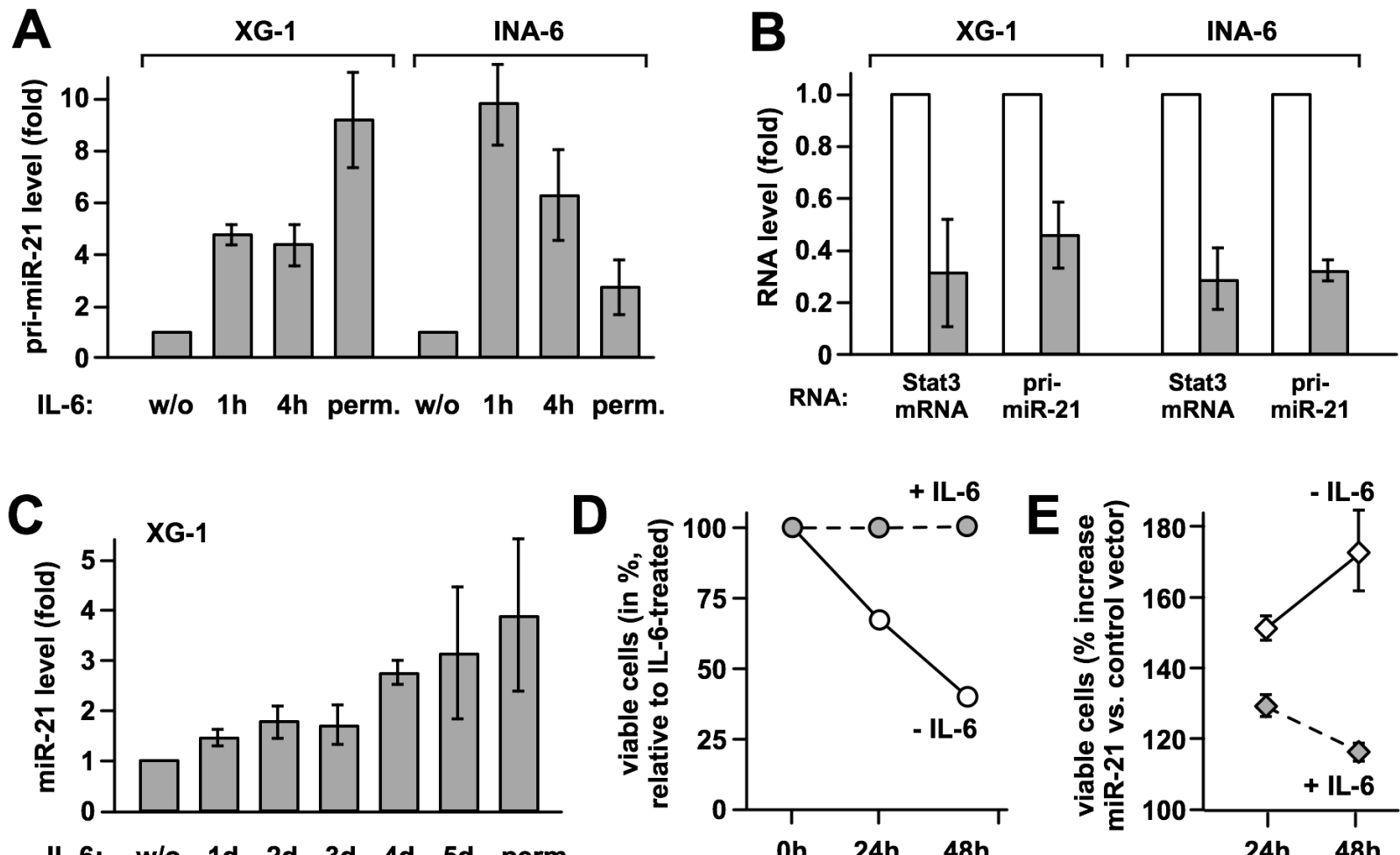

IL-6: w/o 1d 2d 3d 4d 5d perm.
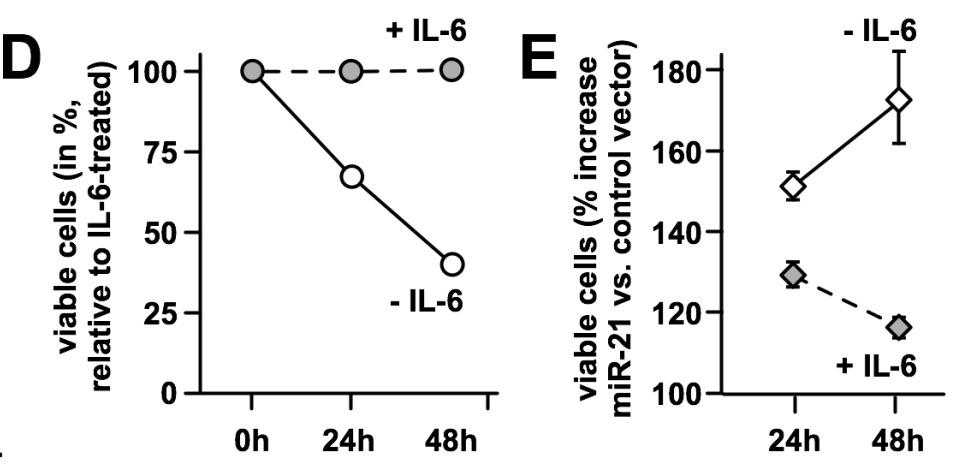

Fig. 2. miR-21 is induced by IL-6 via Stat3 activation and promotes survival of myeloma cells. (A) XG-1 and INA-6 cells were either restimulated with IL- 6 for the times indicated after cytokine withdrawal for 72 and 12 h, respectively, or continuously cultured with IL-6 (perm.). Levels of pri-miR-21 were determined by real-time PCR. Values obtained for cells deprived of IL-6 were set to 1. (B) XG-1 and INA-6 cells cultured in the presence of IL-6 were transiently transfected with an expression plasmid for a small hairpin RNA silencing Stat3 (grey boxes) or a scrambled sequence RNA (open boxes) together with a vector encoding enhanced green fluorescence protein. Successful downregulation of Stat3 protein levels under these conditions has been demonstrated by us previously. ${ }^{9}$ After 48 h, green fluorescing cells were sorted, and Stat3 and pri-miR-21 transcript levels determined by real-time PCR. Values for the control samples were taken as reference. (C) In XG-1 cells treated as above, mature miR-21 was quantified by stem-loop reverse transcription followed by real-time PCR. ${ }^{10}$ (D,E) INA-6 cells were transiently transfected by electroporation with a control (D) or a miR-21 expression vector (E). An expression plasmid encoding enhanced green fluorescent protein was cotransfected. One day posttransfection, cell culture was continued either in the presence or absence of IL-6. After additional 24 or 48 h, apoptosis was studied by flow cytometric Annexin V assay, with the transfected cells gated on the basis of green fluorescence. In (D), the reduced levels of viable (Annexin V-negative) cells after cytokine withdrawal are represented relative to those observed in IL-6-treated cells. Graph E shows the relative percentage of viable cells with miR-21 expression vector relative to control vector transfection. Data represent mean values \pm standard deviation from three independent experiments.

Equivalent observations were made in XG-1 cells (data not shown). In summary, these data provide strong evidence that induction of miR-21 contributes to the antiapoptotic function of Stat3.

Stat3 controls the expression of a number of antiapoptotic proteins on the transcriptional level, including survivin, Bcl-2, and Mcl-1, ${ }^{15-18}$ thereby providing an important fast-track survival 
signal for myeloma and presumably other tumor cells. However, recent data suggest that this regulatory route does not sufficiently explain the full antiapoptotic potential of Stat $3 .^{5,19}$ As demonstrated here, miR-21 induction by Stat3 represents a rather slow-acting yet long-lasting survival stimulus, which appears as an ideal complementation of the immediate induction of antiapoptotic proteins. Overexpression of miR-21 has been reported for numerous cancers, ${ }^{11,20,21}$ all of which contain constitutively activated Stat3 or even rely on Stat3 with respect to cell survival or growth. ${ }^{11,12,15,16,22}$ Taken together, our observations strongly suggest a pivotal relevance of miR-21 for the oncogenic potential of Stat 3 and thereby for its involvement in the pathogenesis of multiple myeloma and other malignancies.

Since miRNAs function as negative regulators of the stability and/or translation of specific target mRNAs, an important step to unravel the molecular mechanism of miR-21 action will be to identify its mRNA targets. Recently, miR-21 was reported to repress the expression of the tumor suppressor PTEN. ${ }^{12}$ Yet, we did not detect upregulation of PTEN expression or AKT phosphorylation in INA-6 and XG-1 cells in response to IL-6 (data not shown).

Stat3 is regarded a promising target for tumor therapy. ${ }^{23-25}$ However, its involvement in the control of numerous cellular processes may cause adverse side effects of such treatments. The identification of miR-21 as a Stat 3 target gene may provide an even more selective and promising target for future therapeutic strategies.

\section{Acknowledgements}

We thank Stefan Rose-John (University of Kiel) for recombinant IL-6, Reuven Agami (The Netherlands Cancer Institute, Amsterdam) for the pSuper vector, and Viola Döbel, Fluorescence Technologies Core Unit of the IZKF Leipzig, for cell sorting. This work was supported in part by the Deutsche Forschungsgemeinschaft, grants numbers SFB610, project C2 (to D.L., C.B., H.C., and F.H.) and BIZ 6-1/3 (to C.F and P.F.S).

\section{Authors}

F.H., K.B.-H., J.H., A.K.K., M.G., and P.F.S designed research, D.L., K.B.-H., G.P., C.F., C.B., K.B., and H.C. performed research, R.B. and M.G. contributed vital new reagents or analytical tools, A.K.U. analysed data, F.H., K.B.-H. and P.F.S wrote the paper.

Conflict-of-interest disclosure: The authors declare no competing financial interests. 


\section{References}

1. Wegenka UM, Buschmann J, Lütticken C, Heinrich PC, Horn F. Acute-phase response factor, a nuclear factor binding to acute-phase response elements, is rapidly activated by interleukin-6 at the posttranslational level. Mol Cell Biol. 1993;13:276-288.

2. Lütticken C, Wegenka UM, Yuan J, et al. Association of transcription factor APRF and protein kinase JAK1 with the IL-6 signal transducer gp130. Science. 1994;263:89-92.

3. Bromberg JF, Wrzeszczynska MH, Devgan G, et al. Stat3 as an oncogene. Cell. 1999;98:295-303.

4. Hodge DR, Hurt EM, Farrar WL. The role of IL-6 and STAT3 in inflammation and cancer. Eur J Cancer. 2005;41:2502-2512.

5. Brocke-Heidrich K, Kretzschmar AK, Pfeifer G, et al. Interleukin-6-dependent gene expression profiles in multiple myeloma INA-6 cells reveal a Bcl-2 family-independent survival pathway closely associated with Stat3 activation. Blood. 2004;103:242-251.

6. Hwang HW, Mendell JT. MicroRNAs in cell proliferation, cell death, and tumorigenesis. $\mathrm{Br}$ J Cancer. 2006;94:776-780.

7. Chen CZ, Li L, Lodish HF, Bartel DP. MicroRNAs modulate hematopoietic lineage differentiation. Science. 2004;303:83-86.

8. Esquela-Kerscher A, Slack FJ. Oncomirs - microRNAs with a role in cancer. Nat Rev Cancer. 2006;6:259-269.

9. Brocke-Heidrich $\mathrm{K}, \mathrm{Ge} \mathrm{B}$, Cvijic H, et al. BCL3 is induced by IL-6 via Stat 3 binding to intronic enhancer HS4 and represses its own transcription. Oncogene. 2006;25:7297-7304.

10. Chen C, Ridzon DA, Broomer AJ, et al. Real-time quantification of microRNAs by stemloop RT-PCR. Nucleic Acids Res. 2005;33:e179.

11. Chan JA, Krichevsky AM, Kosik KS. MicroRNA-21 is an antiapoptotic factor in human glioblastoma cells. Cancer Res. 2005;65:6029-6033.

12. Meng F, Henson R, Lang M, et al. Involvement of human micro-RNA in growth and response to chemotherapy in human cholangiocarcinoma cell lines. Gastroenterology. 2006;130:2113-2129.

13. Si ML, Zhu S, Wu H, Lu Z, Wu F, Mo YY. miR-21-mediated tumor growth. Oncogene. 2006;Epub ahead of print: 10.1038/sj.onc.1210083.

14. Cai X, Hagedorn $\mathrm{CH}$, Cullen BR. Human microRNAs are processed from capped, polyadenylated transcripts that can also function as mRNAs. RNA. 2004;10:1957-1966.

15. Isomoto H, Kobayashi S, Werneburg NW, et al. Interleukin 6 upregulates myeloid cell leukemia-1 expression through a STAT3 pathway in cholangiocarcinoma cells. Hepatology. 2005;42:1329-1338.

16. Konnikova L, Kotecki M, Kruger MM, Cochran BH. Knockdown of STAT3 expression by RNAi induces apoptosis in astrocytoma cells. BMC Cancer. 2003;3:23.

17. Aoki Y, Feldman GM, Tosato G. Inhibition of STAT3 signaling induces apoptosis and decreases survivin expression in primary effusion lymphoma. Blood. 2003;101:1535-1542.

18. Jourdan M, Veyrune JL, Vos JD, Redal N, Couderc G, Klein B. A major role for Mcl-1 antiapoptotic protein in the IL-6-induced survival of human myeloma cells. Oncogene. 2003;22:2950-2959.

19. Tumang JR, Hsia CY, Tian W, Bromberg JF, Liou HC. IL-6 rescues the hyporesponsiveness of c-Rel deficient B cells independent of Bcl-xL, Mcl-1, and Bcl-2. Cell Immunol. 2002;217:47-57.

20. Volinia S, Calin GA, Liu CG, et al. A microRNA expression signature of human solid tumors defines cancer gene targets. Proc Natl Acad Sci USA. 2006;103:2257-2261. 
21. Iorio MV, Ferracin M, Liu CG, et al. MicroRNA gene expression deregulation in human breast cancer. Cancer Res. 2005;65:7065-7070.

22. Rahaman SO, Harbor PC, Chernova O, Barnett GH, Vogelbaum MA, Haque SJ. Inhibition of constitutively active Stat 3 suppresses proliferation and induces apoptosis in glioblastoma multiforme cells. Oncogene. 2002;21:8404-8413.

23. Klampfer L. Signal transducers and activators of transcription (STATs): Novel targets of chemopreventive and chemotherapeutic drugs. Curr Cancer Drug Targets. 2006;6:107-121.

24. Haura EB, Turkson J, Jove R. Mechanisms of disease: Insights into the emerging role of signal transducers and activators of transcription in cancer. Nat Clin Pract Oncol. 2005;2:315-324.

25. Secko D. Cancer therapy finds a solid target. CMAJ. 2005;173:246. 